\title{
Thermal decomposition of chemically treated cellulosic fibers
}

\author{
Emma Jakab $^{1 *}$, Ágnes Bora ${ }^{2}$, Zoltán Sebestyén ${ }^{1}$, Judit Borsa ${ }^{2,3}$ \\ ${ }^{1}$ Institute of Materials and Environmental Chemistry, Research Centre for Natural Sciences, Hungarian \\ Academy of Sciences, 1117 Budapest, Hungary \\ ${ }^{2}$ Faculty of Chemical Technology and Biotechnology, Budapest University of Technology and Economics, \\ 1111 Budapest, Hungary \\ ${ }^{3}$ Rejtő Sándor Faculty of Light Industry and Environmental Engineering, Óbuda University, 1034 Budapest, \\ Hungary \\ *E-mail: jakab.emma@ttk.mta.hu
}

\begin{abstract}
The thermal decomposition of cotton and hemp fibers were studied after mild alkaline treatments with tetramethyl-, tetraethyl-, and tetrabutylammonium hydroxides with the goal of modeling the chemical activation during carbonization of cellulosic fibers. The thermal decomposition was studied by thermogravimetry/mass spectrometry and pyrolysis-gas chromatography/mass spectrometry (Py-GC/MS). The treated samples decomposed in two temperature ranges during heating in the thermobalance. At lower temperature, tetraalkylammonium hydroxides (TAAH) ionically bonded to the cellulose molecules were decomposed; moreover, the alkaline agents initiated the partial decomposition of cellulose. Those fiber segments, which were not accessible for TAAH, decomposed at similar temperatures as the original cotton and hemp samples. It is known that quaternary ammonium hydroxides swell the cellulosic fibers; however, the results of this study proved that there was a chemical interaction between the alkaline swelling agents and cotton or hemp fibers at rather low temperatures $\left(200-300{ }^{\circ} \mathrm{C}\right)$. The evolved products indicated that the alkaline chemicals reacted with the cellulose molecules and alkylated compounds were formed. This observation was confirmed by thermochemolysis experiments carried out by Py-GC/MS using tetramethylammonium hydroxide reagent. The thermochemolysis experiments under mild conditions resulted in the methylation of the glucoside units and levoglucosan, and no peeling reactions of the sugar units was observed as during strong alkaline conditions described in the literature.
\end{abstract}

Keywords Cellulose, Biomass, Tetraalkyl ammonium hydroxide, TMAH, Carbonization, Pyrolysis, Thermogravimetry

$\begin{array}{ll}\text { Abbreviations } & \\ \text { TG } & \text { Thermogravimetric curve } \\ \text { DTG } & \text { Differential thermogravimetric curve } \\ T_{\text {peak }} & \text { Temperature belonging to the maximum of the }-\mathrm{d} m / \mathrm{d} t \text { curves } \\ \text { TG/MS } & \text { Thermogravimetry/mass spectrometry } \\ \text { Py-GC/MS } & \text { Pyrolysis-gas chromatography/mass spectrometry } \\ \text { RT } & \text { Retention time } \\ M & \text { Molar mass }\end{array}$


THM

TAAH

TMAH

TEAH

TBAH
Thermally-assisted hydrolysis and methylation

Tetraalkylammonium hydroxide

Tetramethylammonium hydroxide

Tetraethylammonium hydroxide

Tetrabutylammonium hydroxide

\section{Highlights}

TAAH not only swells cellulosic fibers, but it is bonded chemically to the fibers.

TAAH moieties in the fibers decompose at $90{ }^{\circ} \mathrm{C}$ higher temperature than the pure alkaline chemicals.

The treated segments of the fibers release cellulosic products, as well, below $300{ }^{\circ} \mathrm{C}$.

Methylated monomeric compounds are evolved from TMAH-treated fibers during pyrolysis.

\section{Introduction}

Biomass carbonization has been studied extensively using numerous feedstocks and various thermal techniques [1-3]. Carbonization of biomass produces pyrolysis oil, gas and charcoal, which is applied in various fields of the industry [3, 4]. Wood charcoal is used as a reductant in the metallurgical industry (mainly steel and ferrosilicon industry) [5]. Charcoal is among the most important renewable fuels (industrial and cooking fuel, syngas feedstock, and pyrotechnics). The term biochar refers to the black carbon produced from any biomass feedstock and used as a soil amendment to improve the agricultural soil fertility [6, 7] and applied for the pollution remediation [8].

Activated carbon is widely used to remove pollutants from gaseous and liquid materials. Activated carbon can be produced by physical or chemical activation [9]. During the physical activation, the biomass is carbonized at first and then the charcoal is activated [10]. Carbonization of biomass is carried out at high temperature in an inert atmosphere to remove the volatile matter and hence to reduce the oxygen content. The formed charcoal is then partially gasified at high temperature using steam or carbon dioxide to increase the pore volume and the surface area of the product.

In the chemical activation process generally one heat-treatment is applied, and the pore volume and surface area are enhanced by the use of impregnated catalysts (e.g., phosphoric acid, zinc chloride, sodium hydroxide) [11]. This process can also be performed in two stages. Dobele et al. [12,13] used phosphoric acid as a dehydration catalyst in the first step and sodium hydroxide activator in the second step.

The carbonization of cellulosic fibers results in charcoals of fibrous character, which can be suitable for filtration and the removal of contaminants from waste water. Various fibrous plant materials have been suggested for the preparation of activated carbon [9, 14]. In this work, cotton and industrial hemp fibers were used for the preparation of activated carbon using chemical activation during the carbonization process.

The thermal decomposition of cellulose [15-18] and various cellulosic fibers [19-24] has been studied for a long time, yet the decomposition mechanism is not fully clarified. The decomposition reactions of cellulose are generally divided into two groups [25-27]. The fragmentation reactions produce low molecular mass products 
(gases, water, aldehydes etc.) and char [28, 29], while the depolymerization reaction results in the formation of levoglucosan [30]. The dehydration and rearrangement reactions parallel with the depolymerization generate various pyran and furan derivatives.

The swelling effect of quaternary ammonium hydroxides on cellulose is well-known [31-33]. Bases of a molecular mass less than 150 are only swelling agents, while those of higher molecular mass are solvents for cellulose [31]. Tetramethylammonium hydroxide (TMAH) is a widely used reagent for the combined derivatization and pyrolysis method that can be designated as a thermally-assisted hydrolysis and methylation (THM) process [34]. THM applied with GC/MS detection of the products is especially useful for the structural elucidation of very complex and polar materials [35] such as natural resins, humic substances [36], soils, sediments [37] and lignocellulosic materials [34, 38, 39]. Model compounds were used to clarify the alkaline hydrolysis and methylating reactions of cellulose using TMAH during pyrolysis [40, 41]. The effect of TMAH and sodium hydroxide of low concentrations was studied on the thermal degradation of cellulose by Tanczos et al. [39]. It was concluded that the interaction with cellulose had a stabilizing effect on TMAH decomposition. The alkylating efficiency of various quaternary alkylammonium hydroxides were compared by Lehtonen et al. [36] on a macromolecular humic substance (fulvic acid) using Py-GC/MS and it was concluded that TMAH is the most suitable alkylating agent for the analytical pyrolysis of very complex humic substances, while TEAH and TBAH were not capable to alkylate all the hydroxyl groups.

The goal of this study was to model the carbonization of cellulosic fibers in the presence of activating agents. The chemical activation of cotton and industrial hemp fibers was performed by three tetraalkylammonium hydroxide additives. Small-scale carbonization was carried out using a thermobalance coupled to a mass spectrometer in order to reveal the decomposition mechanism under well-defined conditions. Furthermore, analytical pyrolysis was applied to get more chemical information about the carbonization processes.

\section{Experimental}

\section{Materials}

Two kinds of cellulosic fibers were used, bleached cotton wool containing $>99 \% \alpha$-cellulose and mechanically cleaned industrial hemp. The water solutions of tetramethyl-, tetraethyl- and tetrabutylammonium hydroxide compounds were purchased from Alfa Aesar. The cellulosic fiber samples $(1 \mathrm{~g})$ were treated with $10 \mathrm{~mL} 10 \%$ water solutions of tetraalkylammonium hydroxides, and the samples were filtered and dried at room temperatures.

Methods

$T G / M S$

The TG/MS experiments were performed in a modified Perkin Elmer TGS-2 thermobalance coupled to a Hiden HAL 301/PIC2 quadrupole mass spectrometer. Approximately $4 \mathrm{mg}$ fiber samples were measured in a platinum 
sample pan in argon atmosphere using $140 \mathrm{~mL} \mathrm{~min}^{-1}$ flow rate. Reference experiments were performed with TMAH, TEAH, and TBAH placing $5 \mu \mathrm{L} 10 \%$ water solutions into the sample pan. The thermobalance was purged by argon gas for 45 minutes before the experiments to remove the residual oxygen from the furnace. The samples were heated at a linear rate of $20{ }^{\circ} \mathrm{C} \mathrm{min}-1$ up to $900{ }^{\circ} \mathrm{C}$. A portion of the volatile products was introduced through a heated glass-lined metal capillary into the mass spectrometer, where the ion intensities were monitored using electron impact ionization (EI) with $70 \mathrm{eV}$ electron energy. The mass range of $\mathrm{m} / \mathrm{z}$ 14150 was scanned except the ions of the argon carrier gas.

\section{$P y-G C / M S$}

The pyrolysis experiments were carried out using a Pyroprobe 2000 (Chemical Data Systems, USA) connected on-line to an Agilent 6890/5973 GC/MS (Agilent Technologies, USA). About $0.7 \mathrm{mg}$ samples were placed into the quartz tube of the pyrolyzer and were heated fast to $550{ }^{\circ} \mathrm{C}$ and kept there for $20 \mathrm{~s}$. The carrier gas was helium, which flushed the volatile products onto the gas chromatographic column. The separation of the products was performed on an Agilent DB-1701 column (30m x $0.25 \mathrm{~mm}$ I.D. x $0.25 \mu \mathrm{m}$ film thickness) held at $40{ }^{\circ} \mathrm{C}$ for $4 \mathrm{~min}$, then heated at a $6{ }^{\circ} \mathrm{C} \mathrm{min}^{-1}$ rate to $280{ }^{\circ} \mathrm{C}$ and kept there for $7 \mathrm{~min}$. The mass spectrometer operated in EI mode with $70 \mathrm{eV}$ and the mass range of 14-500 Da was scanned.

THM experiments were performed on $1 \mathrm{mg}$ cotton fiber applying $10 \mu \mathrm{L} 10 \%$ solution of TMAH. After drying, the sample was pyrolyzed at $550{ }^{\circ} \mathrm{C}$ for $20 \mathrm{~s}$. This sample is named fresh-treated cotton. The parameters of GC/MS analysis were the same as for the other samples.

\section{Results and discussions}

Thermal decomposition of the untreated cotton and hemp samples

The thermal decomposition of cotton wool occurred in the temperature range between 300 and $420{ }^{\circ} \mathrm{C}$ as shown in Figure 1. 

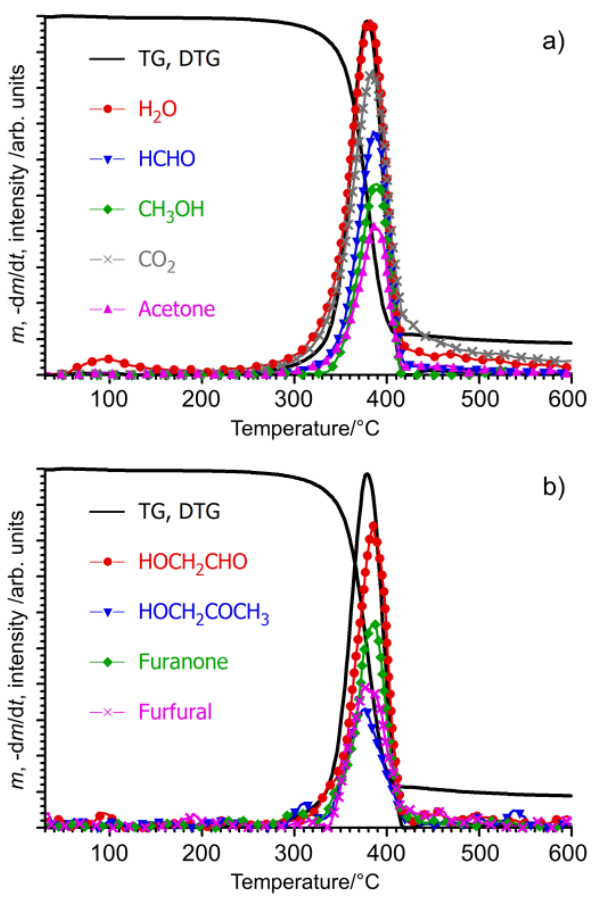

Fig. 1 TG/MS curves of untreated cotton. TG, DTG and the evolution profiles of a few important products: $\mathrm{m} / \mathrm{z}$ 18, water; $m / z$ 30, formaldehyde; $m / z, 32$, methanol; $m / z 44$, carbon dioxide; $m / z 58$, acetone; $m / z 60$, hydroxyacetaldehyde; $m / z 74$, hydroxypropanone; $m / z 84$, furanone; $m / z 96$, furfural

The maximal decomposition rate took place at $378{ }^{\circ} \mathrm{C}$ and the char residue determined at $900{ }^{\circ} \mathrm{C}$ was $8.1 \%$ as presented in Table 1. The cotton sample contained adsorbed water, which evaporated mostly at room temperature before the experiment during purging the thermobalance by dry argon gas. The residual moisture content (about $1 \%$ ) evaporated at around $100{ }^{\circ} \mathrm{C}$ as confirmed by the mass spectrometric curve of water (Fig. 1a). Cellulose represents the major component of the cotton sample, which decomposes via various decomposition routes $[15,26,27]$. One group of the reactions is fragmentation reactions producing gases, water, and char. Fig. 1a presents the most significant low-molecular mass products measured by TG/MS. Water, formaldehyde and methanol were probably derived from the cleavage of the hydroxyl and hydroxymethylene side groups of cellulose molecules. The scission of the glucopyranose rings led to the formation of other oxocompounds, such as acetone (Fig. 1a), hydroxyacetaldehyde, and hydroxypropanone (Fig. 1b). The formation of carbon dioxide can be explained by the oxidation of C-O groups. The other type of reactions is the depolymerization of the carbohydrate chains producing primarily levoglucosan (1,6-anhydro- $\beta$-Dglucopyranose). We could not detect levoglucosan by TG/MS due to its high polarity and the condensation in the transfer line. The dehydration and the rearrangements of the glucopyranose rings resulted in the formation of furan derivatives, as the evolution of furanone and furfural illustrates in Fig. $1 \mathrm{~b}$. 
Table 1 Effect of various treatments on the temperature of maximal decomposition rate and the char yield during the thermal decomposition of cotton and hemp

\begin{tabular}{lcccc}
\hline Samples & \multicolumn{2}{c}{ Cotton } & \multicolumn{2}{c}{ Hemp } \\
\hline Untreated & $T_{\text {peak }} /{ }^{\circ} \mathrm{C}$ & $\begin{array}{c}\text { Char yield* } \\
/ w \%\end{array}$ & $T_{\text {peak }} /{ }^{\circ} \mathrm{C}$ & $\begin{array}{c}\text { Char yield* } \\
/ w^{*}\end{array}$ \\
Washed & 378 & 8.1 & 366 & 12.7 \\
TMAH-treated & 370 & 5.4 & 363 & 13.3 \\
TEAH-treated & 369 & 16.8 & 347 & 12.2 \\
TBAH-treated & 380 & 13.4 & 367 & 13.1 \\
\hline
\end{tabular}

* Determined at $900{ }^{\circ} \mathrm{C}$.

The thermal decomposition of hemp fiber proceeded similarly as that of cotton fiber. About $2 \%$ moisture remained in hemp after the purging period, as the TG curve and the evolution of water demonstrates in Fig. 2 a. The decomposition of the major component, cellulose occurred at about $10^{\circ} \mathrm{C}$ lower temperature than in case of cotton as the $T_{\text {peak }}$ data indicate in Table 1 . The char yield was $12.7 \%$, which is significantly higher than that of cotton.
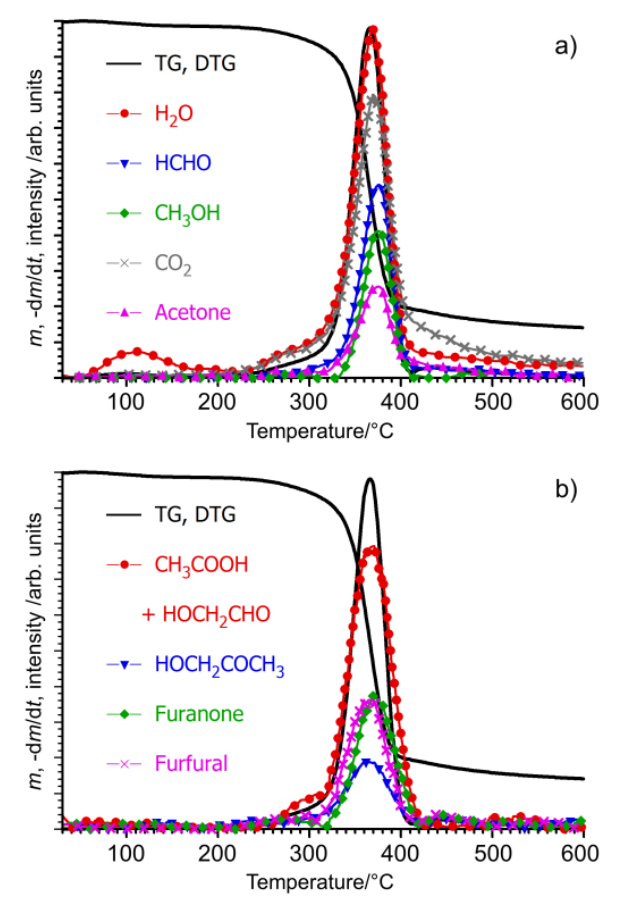

Fig. 2 TG/MS curves of untreated hemp. TG, DTG and the evolution profiles of a few important products: $\mathrm{m} / \mathrm{z}$

18, water; $m / z$ 30, formaldehyde; $m / z$ 32, methanol; $m / z$ 44, carbon dioxide; $m / z$ 58, acetone; $m / z$ 60, acetic acid and hydroxyacetaldehyde; $m / z, 74$, hydroxypropanone; $m / z, 84$, furanone; $m / z, 96$, furfural

The increased char yield can be explained by the lignin content of hemp, which produces large amount of char. The decomposition started at lower temperature (at about $250{ }^{\circ} \mathrm{C}$ ) than in case of cotton (at about $300{ }^{\circ} \mathrm{C}$ ), 
which can be due to the lignin and the hemicellulose content of hemp. The evolution curves of water and carbon dioxide confirm the decomposition of these components above $250{ }^{\circ} \mathrm{C}$ (Fig. 2a).

Furthermore, the evolution profile of the $\mathrm{m} / \mathrm{z} 60$ ions also shows a peak at about $300{ }^{\circ} \mathrm{C}$ (Fig. $2 \mathrm{~b}$ ), which can be attributed to the release of acetic acid from the acid groups of hemicellulose and lignin. The main peak of the $\mathrm{m} / \mathrm{z} 60$ ion curve at about $370{ }^{\circ} \mathrm{C}$ represents hydroxyacetaldehyde, which is one of the main fragmentation products of cellulose. Although the $\mathrm{m} / \mathrm{z} 60$ ions represent both acetic acid and hydroxyacetaldehyde, the identification is possible on the basis of the characteristic fragment ions, $m / z 45$ and 31 , respectively. The decomposition of cellulose component of hemp proceeds similarly to that of cotton, but the intensity of the higher molecular mass products is lower in agreement with the lower cellulose content.

Thermal decomposition of quaternary ammonium hydroxides

Before studying the interaction between the quaternary ammonium hydroxides and the cellulosic fibers, the thermal decomposition of tetraalkylammonium hydroxides has been analyzed by TG/MS. Figure 3 shows the TG/MS plots of tetramethyl- and tetraethylammonium hydroxide. The decomposition of tetraethyl- and tetrabutylammonium hydroxide is quite similar; therefore the decomposition of the former is only presented here. As Fig. 3a demonstrates, TMAH released about $20 \%$ water below $100{ }^{\circ} \mathrm{C}$, which originated apparently from the crystalline water bonded to TMAH. It is likely that the water solvent was released during purging the furnace before the experiment. The decomposition of TMAH proceeded by dissociation reactions leading to the formation of trimethylamine and methanol between 100 and $200{ }^{\circ} \mathrm{C}$, as the evolution profiles of the molecular ions ( $m / z 59$ and 32) indicate in Fig. 3a. The decomposition of TEAH occurred at about $50{ }^{\circ} \mathrm{C}$ lower temperature with the evolution of triethylamine and ethanol. Fig. $3 \mathrm{~b}$ shows the molecular ion of triethylamine $(\mathrm{m} / \mathrm{z}$ 101) and the main fragment ion of ethanol $(\mathrm{m} / \mathrm{z} 31$, hydroxymethyl) since the molecular ion $(\mathrm{m} / \mathrm{z} 46)$ has very low intensity in the mass spectrum of ethanol. None of the tetraalkylammonium hydroxides produced char residue indicating that no secondary reactions took place during decomposition. 

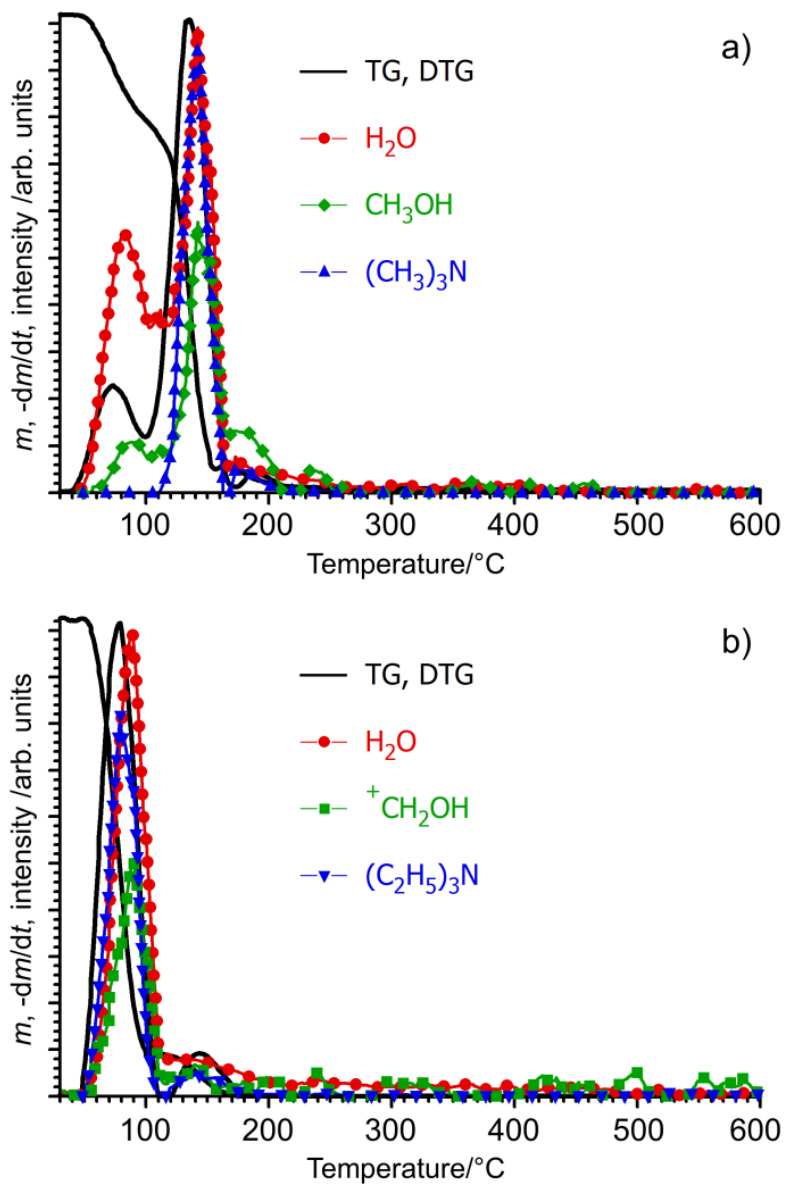

Fig. 3 TG/MS curves of (a) TMAH and (b) TEAH. TG, DTG and the evolution profiles of the products: $\mathrm{m} / z$ 18, water; $m / z$ 31, hydroxymethyl fragment ion; $m / z$ 32, methanol; $m / z$ 59, trimethylamine; $m / z, 101$, triethylamine

Effect of quaternary ammonium hydroxides on the thermal behavior of cotton and hemp

Figure 4 presents the thermogravimetric curves of the untreated and treated cotton samples. As the tetraalkylammonium hydroxide treatments were performed in water solution, a reference sample was prepared using pure water, as well. TG (Fig. 4a) and DTG (Fig. 4b) curves show that the difference between the thermal behavior of untreated and water treated (washed) samples is rather small. The peak temperature of the DTG curve is only $8{ }^{\circ} \mathrm{C}$ lower (Table 1 ); however, the char yield reduced by $2.7 \%$ that can be explained by the removal of some impurities from the cotton fiber. 

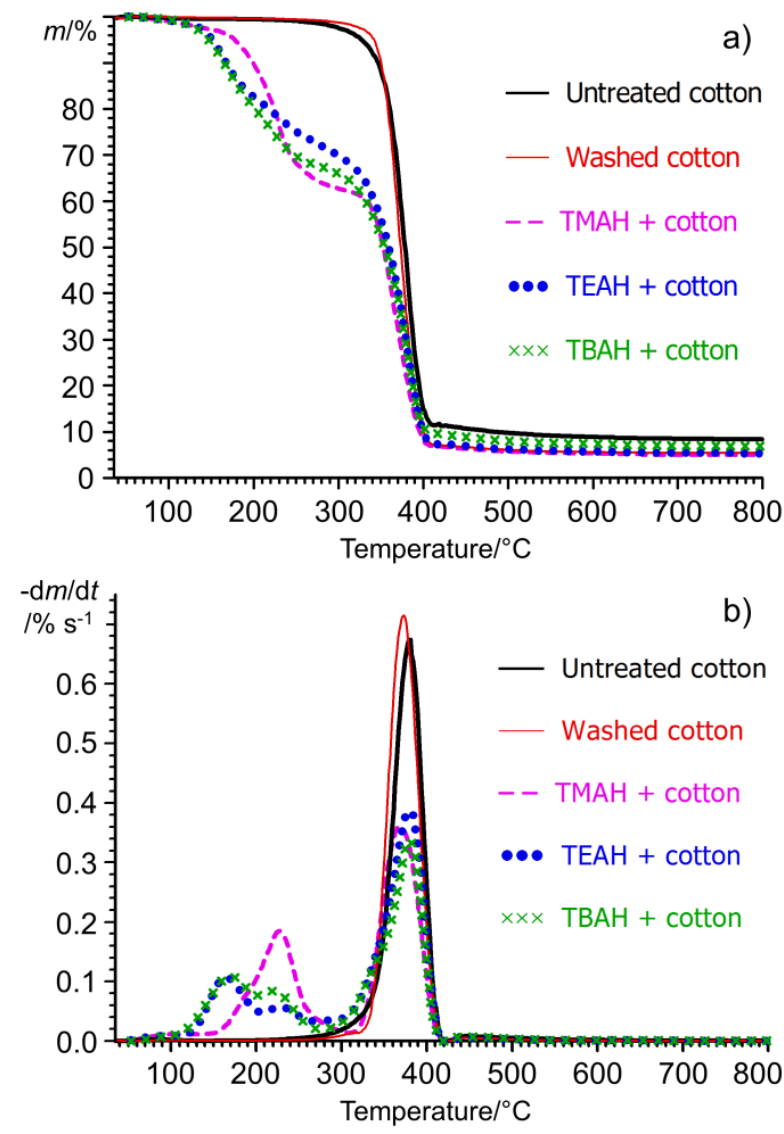

Fig. 4 (a) TG and (b) DTG curves of untreated and treated cotton

The thermal decomposition of the alkali treated cotton samples occurred in two main stages (Fig. 4). The lowtemperature mass loss below $300{ }^{\circ} \mathrm{C}$ (about 30-35\%) can be attributed mainly to the release of alkaline chemicals bonded to the cellulose fibers. Cellulose molecules degraded in the main devolatilization step between 300 and $420{ }^{\circ} \mathrm{C}$, similarly to the untreated and washed cotton samples. The largest difference between the decomposition temperatures ( $T_{\text {peak }}$ ) of all cotton samples is only $11{ }^{\circ} \mathrm{C}$ (Table 1$)$. It can be established that there is an interaction between the alkaline chemicals and the cotton fibers because the formation of volatile products occurred at about $90{ }^{\circ} \mathrm{C}$ higher temperature than the decomposition of the pure chemicals (Fig. 3). TMAH-treated segments of cotton were thermally more stable than TEAH- and TBAH-treated cotton segments, which is in agreement with the stability of the pure alkaline chemicals. The maximum of the DTG curve is 230 ${ }^{\circ} \mathrm{C}$ for the first decomposition step of TMAH-treated cotton and it is $160{ }^{\circ} \mathrm{C}$ for TEAH- and TBAH-treated cotton. It indicates that the interaction of cellulose is stronger with the smaller TMAH molecules than with the larger TEAH and TBAH molecules. The more compact TMAH molecules probably were able to penetrate into the non-polar intermolecular sheets of cellulose as it was discussed by Tóth et al. [33].

As Figure 5 and Table 1 illustrate, the washed and untreated hemp samples decomposed very similarly due to the cleaning of hemp fibers before use. Hemp sample absorbed higher amount of quaternary ammonium hydroxides than cotton as the comparison of Figures 4 and 5 indicates. The mass loss up to $300{ }^{\circ} \mathrm{C}$ amounts to $50-60 \%$ of the alkaline-treated samples. The DTG curves of TEAH- and TBAH-treated hemp show that the first decomposition step involves two processes suggesting that there are weaker and stronger interactions between 
the chemicals and hemp. The second peak or shoulder coincides with the decomposition of TMAH-treated hemp. Above $300{ }^{\circ} \mathrm{C}$, apparently the residual hemp fibers degraded with $T_{\text {peak }}$ temperatures between 347 and $367^{\circ} \mathrm{C}$.

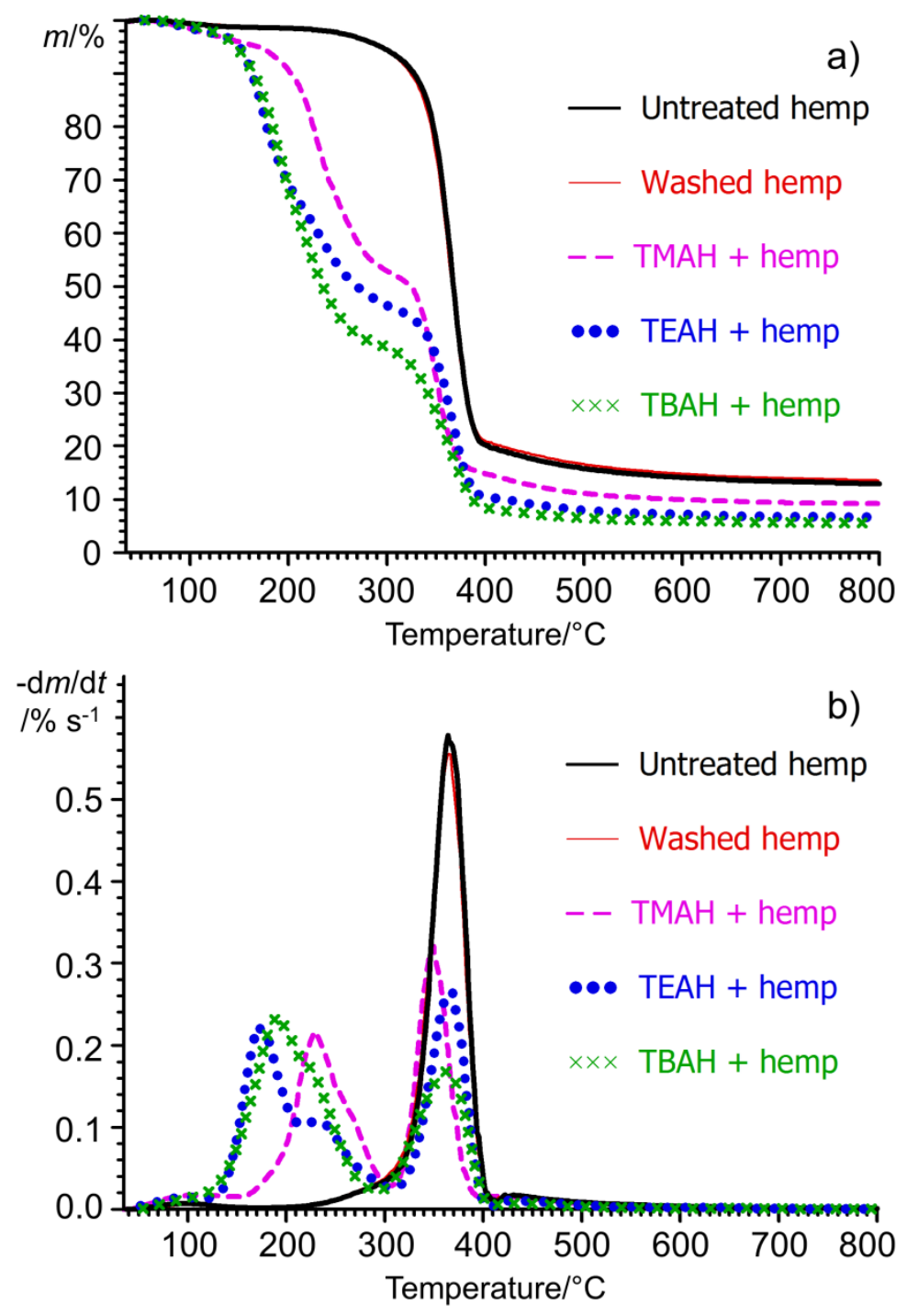

Fig. 5 (a) TG and (b) DTG curves of untreated and treated hemp

Decomposition products of TMAH-treated fibers

The thermal decomposition of the quaternary alkylammonium hydroxide-treated samples proceeded similarly, therefore the TG/MS curves of TMAH-treated samples are only presented here. Figure 6 shows the evolution profiles of a few characteristic compounds produced during the thermal decomposition of TMAH-treated cotton (Fig. 6a) and hemp (Fig. 6b). TMAH was applied to the fibers from water solutions, hence the amount of adsorbed water increased in both samples significantly in comparison with the untreated samples (Figs. 1a, 2a, and 6). After the evaporation of the moisture content, probably the crystalline water bonded to TMAH residues was released at about $200{ }^{\circ} \mathrm{C}$, since the crystalline water of TMAH is released just before the decomposition of TMAH [39]. The water evolution peak at about $240{ }^{\circ} \mathrm{C}$ accompanies the decomposition of the TMAH-treated 
segments, while the main water peak can be attributed to the scission of the hydroxyl functional groups of cellulose in cotton and hemp at about 370 and $350{ }^{\circ} \mathrm{C}$, respectively.
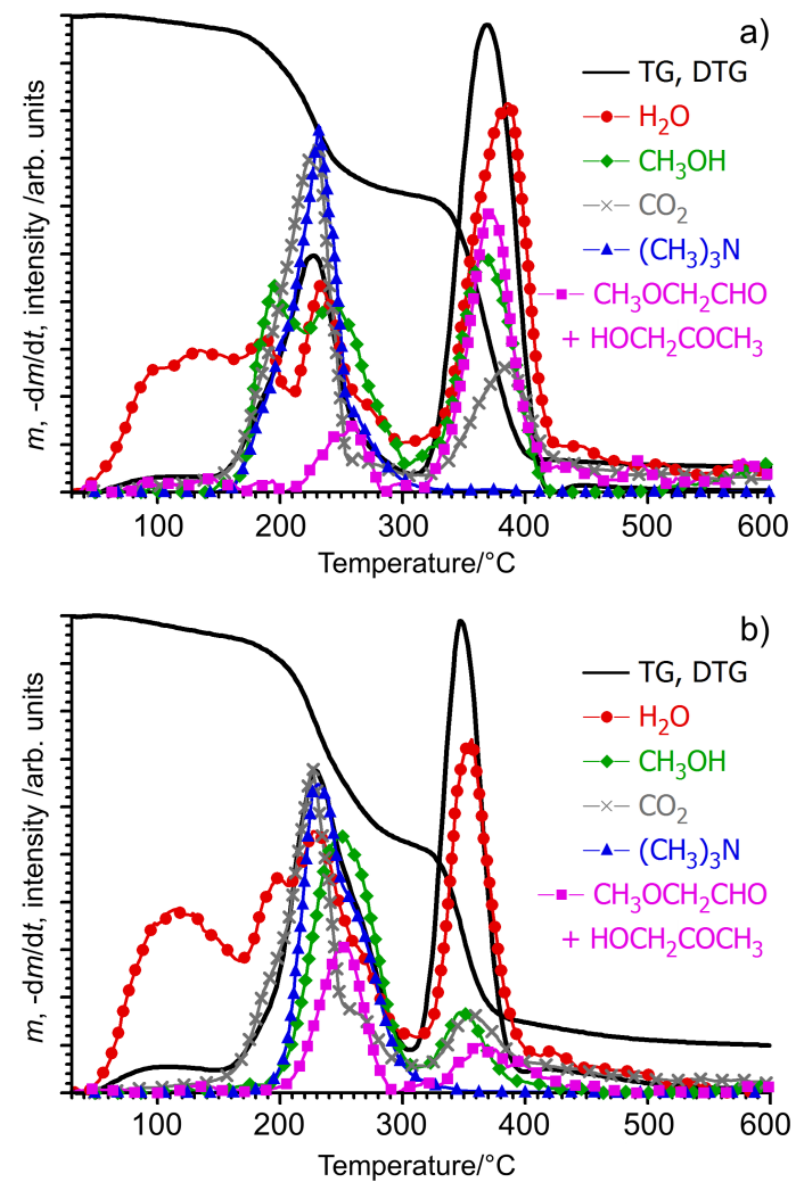

Fig. 6 TG/MS curves of (a) TMAH-treated cotton and (b) TMAH-treated hemp. TG, DTG and the evolution profiles of a few important products: $m / z$ 18, water; $m / z$ 32, methanol; $m / z$ 44, carbon dioxide; $m / z$ 59, trimethyl amine; $m / z$ 74, 2-methoxyacetaldehyde and hydroxypropanone

The release of carbon dioxide from TMAH-treated fibers started at about $150{ }^{\circ} \mathrm{C}$, when the temperature is increased in the thermobalance. Its formation indicates the oxidation of cellulose in the presence of the alkaline swelling agents; for example, the terminal $-\mathrm{CH}_{2} \mathrm{OH}$ groups might have been oxidized to produce $\mathrm{CO}_{2}$. $\mathrm{The}$ devolatilization of TMAH residues occurred between 150 and $300{ }^{\circ} \mathrm{C}$; however, the evolution curves of various compounds did not coincide indicating that more decomposition reactions took place in this temperature range. Pure TMAH decomposed by the simultaneous release of trimethylamine and methanol (Fig. 3a); however, the formation of these products occurred at different temperatures from the TMAH-treated fibers (Fig. 6). This observation proves that chemical reaction took place between TMAH and the fibers. Trimethylamine evolution was centered at about $230{ }^{\circ} \mathrm{C}$ from both samples, although cotton started to release it at a lower temperature, while the evolution curve of hemp has a shoulder at higher temperature. It means that tetramethylammonium ions are bonded to various sites, which have slightly different thermal stability. Methanol formation occurred at about $250{ }^{\circ} \mathrm{C}$ from both samples; however, TMAH-treated cotton released methanol in a separated peak at about $200{ }^{\circ} \mathrm{C}$, as well. TMAH treatment caused methylation of certain cellulose moieties during thermal 
decomposition, and these reactions took place at about $100{ }^{\circ} \mathrm{C}$ lower temperature than the decomposition of pristine cellulose. It is proved by the formation of methoxyacetaldehyde at about $250{ }^{\circ} \mathrm{C}$ from both samples. Hydroxyacetaldehyde is a typical product of cellulose formed by the fragmentation of the glucopyranose rings between 320 and $420{ }^{\circ} \mathrm{C}$, as shown in Figs. $1 \mathrm{~b}$ and $2 \mathrm{~b}$. Its methylated derivative, methoxyacetaldehyde was formed from the TMAH-treated samples at as low temperature as $200-300{ }^{\circ} \mathrm{C}$. Apparently not only the original hydrogen-bonded microcrystalline structure of cellulose was disrupted during swelling in the alkaline chemicals, but chemical reactions took place between TAAH and the fibers. Higher yield of methoxyacetaldehyde was formed from hemp (Fig. 6b) than from cotton (Fig. 6a), which indicates the higher methoxy content of hemp. As discussed above, the hemp sample absorbed higher amount of TMAH than cotton and hence more methoxy groups were formed in hemp.

Cellulose segments that were not accessible for the alkaline agents decomposed above $300{ }^{\circ} \mathrm{C}$ similarly as the original cotton and hemp samples. There was no trimethylamine evolution in this temperature range confirming that the residues of the alkylating agents decomposed below $320{ }^{\circ} \mathrm{C}$. Hemp took up higher amount of TMAH and released more volatiles in the first decomposition range, therefore it produced lower amount of volatile products in the second decomposition stage than TMAH-treated cotton. Figure 6 shows the evolution curve of the $\mathrm{m} / \mathrm{z} 74$ ions, which represent the molecular ions of two products, methoxyacetaldehyde and hydroxypropanone. Their identification was based on the pyrolysis-GC/MS results, as discussed below. Methoxyacetaldehyde was evolved between 200 and $300^{\circ} \mathrm{C}$, as detailed above. Hydroxypropanone was formed from the cellulose chains during the fragmentation of the pyranose rings. Its yield was higher from cotton because the cellulose content of cotton fibers is higher than that of hemp.

Effect of TMAH treatment on the pyrolysis products of cotton

Pyrolysis-GC/MS experiments have been carried out on the untreated and treated samples in order to get more information on the product distributions and the mechanisms. The TG results indicated that the decomposition processes were ended by $550^{\circ} \mathrm{C}$, therefore this was chosen as the pyrolysis temperature. Figure 7 presents the pyrolysis-gas chromatograms (pyrograms) of untreated and TMAH-treated cotton samples as examples for the pyrolytic behavior of cellulosic fibers. In addition to the pyrolysis of the previously treated TMAH-cotton sample, we carried out another experiment for comparison, where TMAH was added to the cotton sample just before the pyrolysis (fresh TMAH-treated sample). Fig. 7a shows the pyrogram of the untreated cotton wool, and Table 2 lists the identification of the major compounds. As it is well-known [15], the main decomposition product of cellulose is levoglucosan (22), which is formed by depolymerization, so it can be considered as the monomer of cellulose. The release of 1,6-anhydro- $\beta$-D-glucofuranose (23) proves that transformation of the pyranose rings occurs into furanose rings, to some extent. The rearrangement and dehydration reactions of the pyranose units lead to the evolution of furan derivatives $(\mathbf{1 1}, \mathbf{1 6})$. The dehydration of the monomeric units results in the formation of a few bicyclo-compounds (13-15) and pyran derivative (19). Hydroxyacetaldehyde $(8)$ and other oxo-compounds $(6,9,10)$ are formed during the scission of the pyranose rings of cellulose.

The TMAH-treated cotton sample (Fig. 7b) released large amounts of trimethylamine (3) and methanol (4) originating from TMAH. Besides gases and water, levoglucosan (22) was the most significant product from the 
cellulose molecules. Furthermore, a new peak appeared in the pyrogram, methyl-2-O-methyl- $\alpha$-Dglucopyranoside (20), which was formed by methylation of the pyranoside bond of cellulose and the hydroxyl group at position 2. Trace amount of dimethyl-methyl-glucopyranoside was also formed (18). The pyrogram of the fresh treated cotton sample (Fig. 7c) shows more methylated compounds, which are methyl-Dglucopyranoside (21), its methylated derivatives $(\mathbf{1 8 , 2 0})$, and dimethyllevoglucosan (17). Furthermore, the pyrogram has two higher molecular mass compounds $(\mathbf{2 4}, \mathbf{2 5})$, which are possibly methylated dimers.
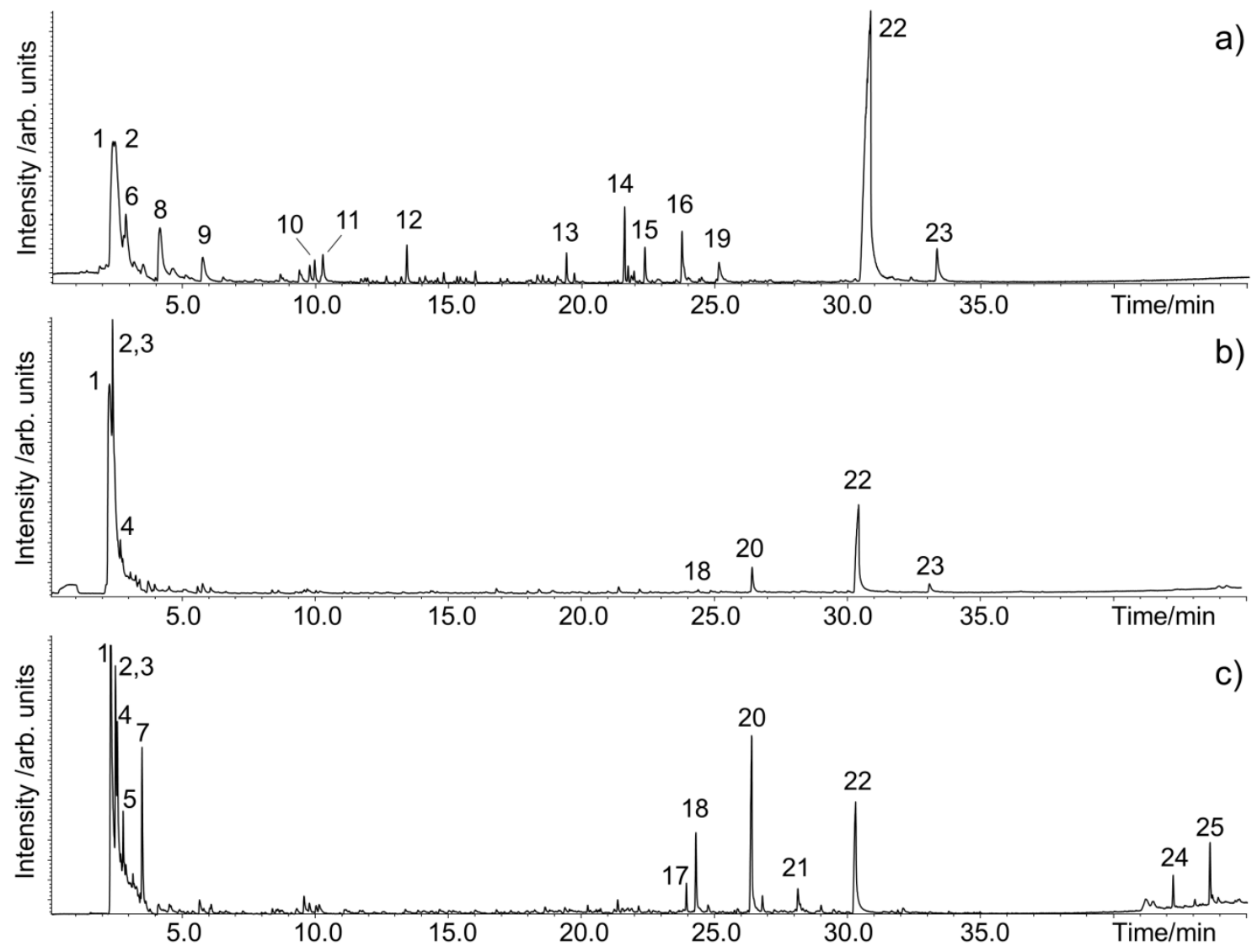

Fig. 7 Pyrolysis gas chromatograms of (a) untreated, (b) TMAH-treated, and (c) fresh TMAH-treated cotton.

The identification of the numbered peaks is found in Table 2 
Table 2 Identification and the main GC/MS characteristics of the pyrolysis products of untreated and TMAHtreated cotton (see Fig. 7)

\begin{tabular}{|c|c|c|c|c|}
\hline No. & $\begin{array}{c}\mathrm{RT} \\
/ \mathrm{min}\end{array}$ & Compound & $\begin{array}{c}M \\
/ \mathrm{g} \mathrm{mol}^{-1}\end{array}$ & Characteristic MS ions \\
\hline 1 & 2.34 & Gaseous products $\left(\mathrm{CO}_{2}, \mathrm{CO}, \mathrm{HCHO}\right)$ & & \\
\hline 2 & 2.41 & Water & 18 & $18,17,16$ \\
\hline 3 & 2.48 & Trimethylamine & 59 & $58,59,42,30$ \\
\hline 4 & 2.55 & Methanol & 32 & $31,32,29,15$ \\
\hline 5 & 2.77 & 2-Propenal & 56 & $56,27,55,29$ \\
\hline 6 & 2.83 & Butanal & 72 & $43,29,15,72$ \\
\hline 7 & 3.48 & Methoxyacetaldehyde & 74 & $45,29,44,74$ \\
\hline 8 & 4.09 & Hydroxyacetaldehyde & 60 & $31,29,32,60,42$ \\
\hline 9 & 5.71 & 1-Hydroxy-2-propanone & 74 & $43,31,74,15$ \\
\hline 10 & 9.91 & 2-Hydroxy-butanal-3-one & 102 & $43,31,102,29$ \\
\hline 11 & 10.23 & 2-Furanaldehyde & 96 & $96,95,39,29,67$ \\
\hline 12 & 13.38 & 1,2-Cyclopentanedione & 98 & $98,55,42,69,41$ \\
\hline 13 & 19.37 & $\begin{array}{l}\text { Levoglucosenone } \\
\text { (6,8-Dioxabicyclo [3.2.1] oct-2-en-4-one) }\end{array}$ & 126 & $98,68,96,39,53$ \\
\hline 14 & 21.57 & (1R)-1-hydroxy-3,6-dioxabicyclo[3.2.1]octan-2-one & 144 & $43,41,69,70,116$ \\
\hline 15 & 22.33 & 1,4:3,6-Dianhydro- $\alpha$-D-glucopyranose & 144 & $69,57,29,41,144$ \\
\hline 16 & 23.73 & 5-Hydroxymethylfurfural & 126 & $97,126,41,39,69$ \\
\hline 17 & 23.95 & Dimethyllevoglucosan & 190 & $101,45,99,127,75,71$ \\
\hline 18 & 24.31 & Methyl-3,6-O-dimethyl-D-glucopyranoside & 222 & $88,87,73,103,45$ \\
\hline 19 & 25.15 & 2-Hydroxymethyl-5-hydroxy-2,3-dihydro-(4H)-pyran-4-one & 144 & $144,87,97,29,57$ \\
\hline 20 & 26.40 & Methyl-2-O-methyl- $\alpha$-D-glucopyranoside & 208 & $74,87,59,57,45$ \\
\hline 21 & 28.13 & Methyl-D-glucopyranoside & 194 & $74,103,73,59,45$ \\
\hline 22 & 30.81 & Levoglucosan & 162 & $60,57,73,29,42$ \\
\hline 23 & 33.27 & 1,6-Anhydro- $\beta$-D-glucofuranose & 162 & $73,69,43,44,61$ \\
\hline 24 & 42.24 & Methylated dimer & & $88,173,45,233,205$ \\
\hline 25 & 43.62 & Methylated dimer & & $88,219,45,173,74,205$ \\
\hline
\end{tabular}

Methylation of the typical fragmentation product, hydroxyacetaldehyde was observed during THM resulting in the formation of methoxyacetaldehyde (7) similarly to the TG/MS experiments. Comparison of the dry and fresh treated samples shows that the methylating effect was significantly higher when TMAH was added to cotton just before the pyrolysis. Although the same mass ratio was applied (TMAH:cotton=1:1) during both treatments, the residual TMAH solution was filtered in the larger scale experiment, while all the solution remained in the pyrolysis tube during the THM experiment, and probably more TMAH was available for the reactions. It is possible that the higher water content of the fresh sample also played role in the pyrolysis and methylation reactions. It should be noted that the formation of saccharinic and isosaccharinic compounds were described in the literature during THM of cellulose [35, 38, 40, 41]; however, we could not detect these products, which can 
be explained by the different objectives and the experimental conditions. The goal of the published studies was to methylate the pyrolysis products completely, and hence very strong alkaline conditions were applied: $25 \%$ TMAH was used in the mass ratio of TMAH:cellulose $\geq 100: 1$. We used much milder conditions and apparently different reactions took place. The glucopyranose rings remained intact during the TMAH treatment, the alkali ions caused no peeling reactions of the sugar units and only partial methylation of the hydroxyl groups occurred. During pyrolysis, transesterification of the ether groups took place: the pyranoside bonds cleaved and the oxygen residues were methylated. Thus, the most important products were methylglucopyranosides.

\section{Conclusions}

Quaternary ammonium hydroxides (methyl, ethyl, and butyl) were used as chemical activating agents in the carbonization process of cotton wool and hemp fiber. The carbonization was modeled using slow heating rate in the TG/MS and high heating rate in the pyrolysis-GC/MS experiments. Significant increase in the char yield was achieved using alkali-treated cotton wool, while the char yield of hemp fiber was not much influenced by the treatments.

Tetraalkylammonium hydroxides decomposed between 50 and $200{ }^{\circ} \mathrm{C}$ with the evolution of trialkylamines and alcohols. TEAH and TBAH were rather labile degrading at about $80^{\circ} \mathrm{C}$, while TMAH decomposed at about 140 ${ }^{\circ} \mathrm{C}$. The treated cotton and hemp fibers presented two main decomposition steps. The first process can be attributed to the elimination of the alkaline chemicals from the fibers by dissociation to amines and alcohols; however, partial decomposition of the treated cellulosic segments occurred, as well. Hydroxyacetaldehyde, the most significant fragmentation product of the pyranose rings, was methylated by TMAH and methoxyacetaldehyde was detected by TG/MS at rather low temperatures between 200 and $300{ }^{\circ} \mathrm{C}$. In the second stage of decomposition, no amine compounds were formed. Those cellulosic fibers, which have not been accessible for the alkaline chemicals, decomposed above $300{ }^{\circ} \mathrm{C}$ similarly to the untreated cotton and hemp samples during the TG/MS experiments.

The higher molecular mass products were analyzed using pyrolysis-GC/MS. The previously TMAH-treated, dried cotton sample produced methylated methylglucopyranoside derivatives in addition to levoglucosan. However, the majority of the methylating agent released methanol and trimethylamine during pyrolysis at 550 ${ }^{\circ} \mathrm{C}$. When the thermochemolysis was carried out on-line in the pyrolyzer, more methylated products were observed probably due to the higher amount of the alkylating agent and the higher water content. Three compounds were identified as methylglucopyranosides, and one compound was formed by the methylation of levoglucosan. Two compounds were observed with rather high molecular mass fragment ions, which were possibly methylated glucose dimers. It was concluded that the alkaline pretreatment with tetramethylammonium hydroxide under mild conditions resulted only in methylation of the glucopyranoside segments and hydroxyacetaldyde, and no peeling reactions of the sugar units took place as described in the literature under strong alkaline conditions.

Acknowledgements This work was supported by NKFIH, Hungary through the Bilateral Cooperation between Hungary and India (project No. TÉT_13_DST-1-2014-0003). Furthermore, the research was carried out 
within project No. VEKOP-2.3.2-16-2017-00013 supported by the European Union and the State of Hungary, co-financed by the European Regional Development Fund. Special thanks are due to T. Tóth, PhD for her valuable advice on cellulose swelling by quaternary ammonium hydroxides.

\section{References}

1. Antal MJ, Jr. Grønli M. The art, science, and technology of charcoal production. Ind Eng Chem Res. 2003;42:1619-40

2. Wang L, Skreiberg O, Van Wesenbeeck S, Gronli M, Antal MJ, Jr. Experimental study on charcoal production from woody biomass. Energy Fuels. 2016;30:7994-8008.

3. Naik DK, Monika K, Prabhakar S, Parthasarathy R, Satyavathi B. Pyrolysis of sorghum bagasse biomass into bio-char and bio-oil products. J Therm Anal Calorim. 2017;127:1277-89.

4. Morales L, Garzón E, Martínez-Blanes JM, Sánchez-Soto PJ. Thermal study of residues from greenhouse crops plant biomass. J Therm Anal Calorim. 2017;129:1111-20.

5. Assis MR, Brancheriau L, Napoli A, Trugilho PF. Factors affecting the mechanics of carbonized wood: literature review. Wood Sci Technol. 2016;50:519-36.

6. Manya JJ. Pyrolysis for biochar purposes: A review to establish current knowledge gaps and research needs. Env Sci Technol. 2012;46:7939-54.

7. Aller MF. Biochar properties: Transport, fate, and impact. Crit Rev Env Sci Technol. 2016;46:1183-96.

8. Ahmad M, Rajapaksha AU, Lim JE, Zhang M, Bolan N, Mohan D, Vithanage M, Lee SS,Ok YS. Biochar as a sorbent for contaminant management in soil and water: A review. Chemosphere 2014;99:19-33.

9. Rashidi NA, Yusup S. A review on recent technological advancement in the activated carbon production from oil palm wastes. Chem Eng J. 2017;314:277-90.

10. Rizhikovs J, Zandersons J, Spince B, Dobele G, Jakab E. Preparation of granular activated carbon from hydrothermally treated and pelletized deciduous wood. J Anal Appl Pyrolysis. 2012;93:68-76.

11. Dobele G, Volperts A, Zhurinsh A, Vervikishko D, Shkolnikov E, Ozolinsh J. Wood based activated carbons for supercapacitor electrodes with sulfuric acid electrolyte. Holzforschung 2017;71:591-8.

12. Dobele G, Vervikishko D, Volperts A, Bogdanovich N, Shkolnikov E. Characterization of the pore structure of nanoporous activated carbons produced from wood waste. Holzforschung 2013;67:587-94.

13. Dobele G, Jakab E, Volperts A, Sebestyén Z, Zhurins A, Telysheva G. Formation of nanoporous carbon materials in conditions of thermocatalytic synthesis. J Anal Appl Pyrolysis. 2013;103:173-80.

14. Ramos ME, Bonelli PR, Cukierman AL, Carrott MMLR, Carrott PJM. Adsorption of volatile organic compounds onto activated carbon cloths derived from a novel regenerated cellulosic precursor. J Hazard Mat. 2010;177:175-82.

15. Shafizadeh F, Bradbury GW. Thermal degradation of cellulose in air and nitrogen at low temperatures. J Appl Polym Sci. 1979;23:1431-1442.

16. Gronli M, Antal MJ,Jr, Várhegyi G. A round-robin study of cellulose pyrolysis kinetics by thermogravimetry, Ind Eng Chem Res. 1999;38:2238-2244. 
17. Molto J, Font R, Conesa JA, Martin-Gullon I. Thermogravimetric analysis during the decomposition of cotton fabrics in an inert and air environment. J Anal Appl Pyrolysis. 2006;76:124-31.

18. Carrier M, Windt M, Ziegler B, Appelt J, Saake B, Meier D, Bridgwater A. Quantitative Insights into the Fast Pyrolysis of Extracted Cellulose, Hemicelluloses, and Lignin. Chemsuschem. 2017;10: in press DOI: $10.1002 /$ cssc. 201700984

19. Meier D, Faix O. State of the art of applied fast pyrolysis of lignocellulosic materials - a review. Biores Technol. 1999;68:71-7.

20. Jakab E, Mészáros E, Borsa J, Effect of slight chemical modification on the pyrolysis behavior of cellulose fibers. J Anal Appl Pyrolysis. 2010;87:117-123.

21. Sebestyén Z, May Z, Réczey K, Jakab E. The effect of alkaline pretreatment on the thermal decomposition of hemp. J Therm Anal Calorim. 2011;105:1061-9.

22. Borsoi C, Zimmernnam MVG, Zattera AJ, Santana RMC, Ferreira CA. Thermal degradation behavior of cellulose nanofibers and nanowhiskers. J Therm Anal Calorim. 2016;126:1867-78.

23. Stevulova N,Estokova A, Cigasova J, Schwarzova I, Kacik F, Geffert A. Thermal degradation of natural and treated hemp hurds under air and nitrogen atmosphere. J Therm Anal Calorim. 2017;128:1649-60.

24. Branca C, Di Blasi C, Galgano A. Experimental analysis about the exploitation of industrial hemp (Cannabis sativa) in pyrolysis. Fuel Proc Technol. 2017;162:20-9.

25. Várhegyi G, Jakab E, Antal MJ,Jr. Is the Broido-Shafizadeh model for cellulose pyrolysis true? Energy Fuels 1994;8:1345-1352.

26. Kawamoto H. Review of reactions and molecular mechanisms in cellulose pyrolysis. Current Org Chem. 2016;20:2444-57.

27. Jakab E. Analytical techniques as a tool to understand the reaction mechanism. In: Pandey EA, Bhaskar T, Stocker M, Kumar Sukumaran R, editors. Recent advances in thermo-chemical conversion of biomass. Elsevier; 2015. pp.73-106.

28. Broido A, Nelson MA. Char yield on pyrolysis of cellulose, Combust Flame. 1975;24:263-268.

29. Piskorz J, Radlein D, Scott D.S. On the mechanism of the rapid pyrolysis of cellulose, J Anal Appl Pyrolysis. 1986;9:121-137.

30. Hosoya T, Sakaki S. Levoglucosan Formation from Crystalline Cellulose: Importance of a Hydrogen Bonding Network in the Reaction. Chemsuschem. 2013;6;2356-68.

31. Krassig H A. Cellulose: Structure, Accessibility and Reactivity. Gordon and Breach Science Publishers; Amsterdam: 1993, pp. 238-277.

32. Tanczos I, Borsa J, László K, Sajó I, Juhász ZA, Tóth T. Effect of tetramethylammonium hydroxide on cotton cellulose compared to sodium hydroxide. Macromol Chem Phys. 2000;201:2550-6.

33. Tóth T, Borsa J, Reiher J, Sallay P, Sajó I, Tanczos I. “Mercerization” of cotton with tetramethylammonium hydroxide. Textile Res J. 2003;73:273-8.

34. Challinor JM. Characterisation of wood by pyrolysis derivatisation-gas chromatography/mass spectrometry. J Anal Appl Pyrolysis. 1995;35:93-107.

35. Shadkami F, Helleur R. Recent applications in analytical thermochemolysis. Review. J Anal Appl Pyrolysis. 2010;89:2-16. 
36. Lehtonen T, Peuravuori J, Pihlaja K. Comparison of quaternary methyl-, ethyl- and butylammonium hydroxides as alkylating reagents in pyrolysis-GC/MS studies of aquatic fulvic acid. J Anal Appl Pyrolysis. 2003;68-69:315-29.

37. Estournel-Pelardy C, Delarue F, Grasset L, Laggoun-Défarge F, Amblès A. Tetramethylammonium hydroxide thermochemolysis for the analysis of cellulose and free carbohydrates in a peat bog. J Anal Appl Pyrolysis. 2011;92:401-6.

38. Fabbri D, Helleur R. Characterisation of the tetramethylammonium hydroxide thermochemolysis products of carbohydrates. J Anal Appl Pyrolysis. 1999;49:277-93.

39. Tanczos I, Pokol G, Borsa J, Tóth T, Schmidt H. The effect of tetramethylammonium hydroxide in comparison with the effect of sodium hydroxide on the slow pyrolysis of cellulose. J Anal Appl Pyrolysis. 2003;68-9:173-85.

40. Schwarzinger C, Tanczos I, Schmidt H. Levoglucosan, cellobiose and their acetates as model compounds for the thermally assisted hydrolysis and methylation of cellulose and cellulose acetate. $\mathrm{J}$ Anal Appl Pyrolysis. 2002;62:179-96.

41. Schwarzinger C. Identification of methylated saccharinolactones and partially methylated saccharinic acids in the thermally assisted hydrolysis and methylation of carbohydrates. J Anal Appl Pyrolysis. 2004;71:501-514. 